\title{
Research Paper: The Effect of Mindfulness-Based Cognitive Therapy on Anxiety of Pre-Hospital Emergency Staff
}

\section{Amin Saberinia ${ }^{1}{ }^{\circ}$, Elaheh Aflatoonian², Forouzandeh Jannat ${ }^{3},{ }^{*}$ Mohsen Aminizadeh ${ }^{4,5}$ 이}

1. Department of Emergency Medicine, School of Health and Safety, Shahid Beheshti Medical University, Tehran, Iran 2. Master of Clinical Psychology, Department of Education in Tehran, Tehran, Iran.

3. Health in Emergency and Disaster, School of Public Health, Tehran University of Medical Sciences, Tehran, Iran

4. Health in Emergency, Disaster Research Center, University of Social Welfare and Rehabilitation Sciences, Tehran, Iran.

5. Department of Health in Emergency, Disaster Research Center, Kerman Medical University, Kerman, Iran.

\begin{tabular}{|c|c|}
\hline $\begin{array}{l}\text { Use your device to scan } \\
\text { and read the article online }\end{array}$ & \\
\hline 回乎乐回 & diftation Saberinia A, Aflatoonian E, Jannat F, Aminizadeh M. [The Effect of Mindfulness-Based Cognitive Therapy on Anxiety \\
\hline Laters: & of Pre-Hospital Emergency Staff (Persian)]. Archives of Rehabilitation. 2020; 20(4):340-349. http://dx.doi.org/10.32598/rj.20.4.340 \\
\hline affis & dol' http://dx.doi.org/10.32598/rj.20.4.340 \\
\hline
\end{tabular}

Received: 04 Jul 2019

Accepted: 27 Nov 2019 Available Online: 01 Jan 2020

Keywords:

Mindfulness-Based

Cognitive Therapy,

Anxiety, Pre-hospital

emergency staff

\section{ABSTRACT}

Objective Anxiety is one of the important problems in the daily lives of medical emergency staff. Experiencing this state and its complications can decrease the quality of work and reduce the desire to continue serving. Mindfulness-Based Cognitive Therapy (MBCT) can be one of the effective interventions for reducing anxiety. The present study aimed to investigate the effect of MBCT on reducing the anxiety of pre-hospital emergency staff.

Materials \& Methods This is quasi-experimental study with pretest-posttest design using control group. The statistical population consisted of all staff of the Center for Disaster Management and Emergency Response in Alborz, Iran in 2017 ( $N=100)$. Of these, 75 were selected who had a moderate to high anxiety score based on the Kettle test. In the end, 30 were entered into the study based on inclusion criteria and then randomly assigned into two groups of control $(n=15)$ and MBCT groups $(n=15)$. The control group received no intervention. Having consent to participate in the study by signing a written form, having no acute physical and psychological disease, not receiving psychological and medication treatment during the past few months and no participation in any other similar studies were the inclusion criteria, while the exclusion criteria were: absence form more than three sessions and having no willingness to continue participation. The experimental group received MBCT for 8 sessions, once per week, each for 90 minutes. The data collection tool was the Cattell's anxiety scale. Collected data were analyzed using descriptive (mean and standard deviation) and inferential statistics (ANCOVA to test the research hypotheses, Kolmogorov-Smirnov to examine the normality of data distribution, and Box's and Levene's tests to test the quality of variances) in SPSS V. 22 software.

Results MBCT could reduce the anxiety of subjects compared to controls ( $F=32.87, P<0.05)$. Moreover, it led to a significant reduction in self-sentiment development $(P<0.05, F=13.32)$ or paranoid trend $(F=9.40$, $\mathrm{P}<0.05)$ in pre-hospital emergency staff. The ANCOVA results showed no significant difference between the MBCT and control groups in terms of guilt proneness, ego-strength, and ergic tension $(P>0.05)$.

Conclusion It was concluded that MBCT can reduce the anxiety of emergency medical staff. It can help them identify anxious thoughts, challenges and struggles with these thoughts, and confronts and replaces them with non-anxious thoughts. MBCT, by reducing their self-sentiment development and paranoid trend, is an effective measure to reduce the anxiety and hence, increase the work efficiency and job satisfaction of pre-hospital emergency personnel and increase their resilience in emergency situations. By improving the MBCT techniques, we can hope that it can improve mental health, quality of work, job satisfaction, and resilience of this medical group.

\section{* Corresponding Author:}

Mohsen Aminizadeh, PhD.

Address: Health in Emergency and Disaster Research Center, University of Social Welfare and Rehabilitation Sciences, Tehran, Iran. Tel: +98 (913) 9456897

E-Mail: mohsen.aminizadeh@gmail.com 


\title{
اثربخشى شناختدرمانى مبتنى بر ذهن آكاهى بر اضطراب كاركنان اورزانس بيشبيمارستانى: يكى مطالعهنيمه تجربى
}

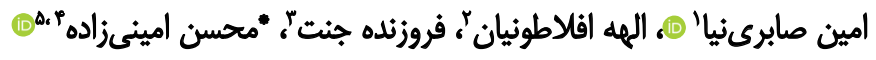 \\ ا. كروه سلامت در بلايا و فوريتهاء دانشكده بهداشت و ايمنى، دانشكاه علوم يزشكى شهيد بهشتى، تهران، ايران.

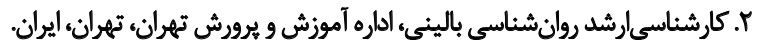

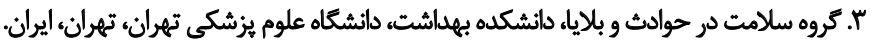

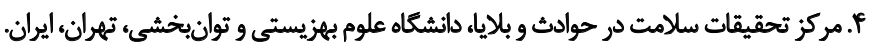

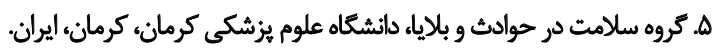

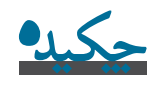

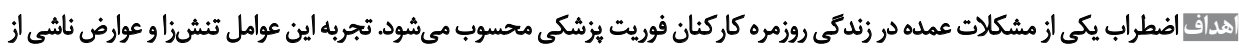

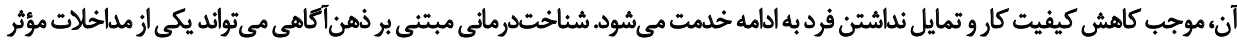

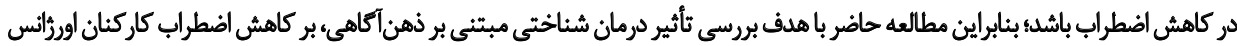

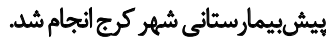

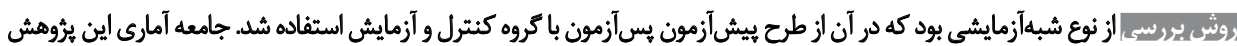

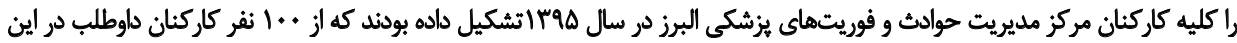

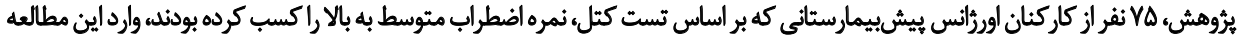

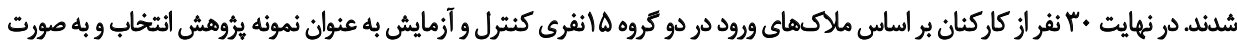

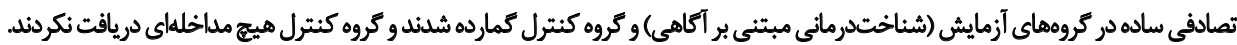

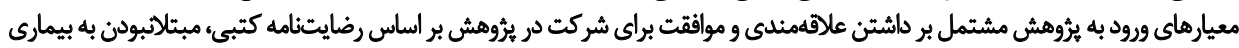

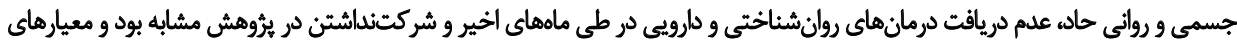

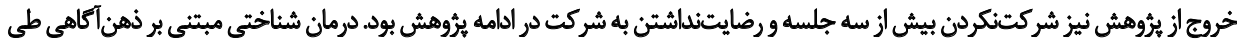

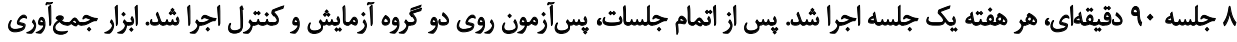

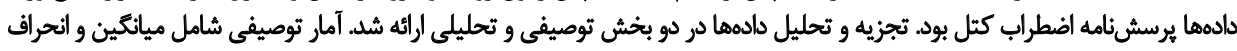

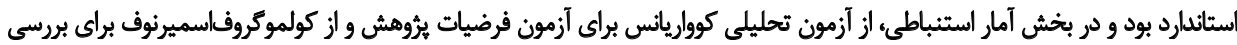

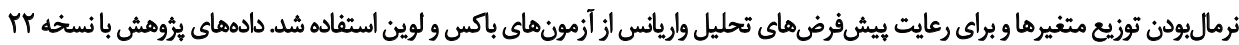

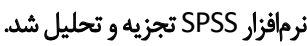

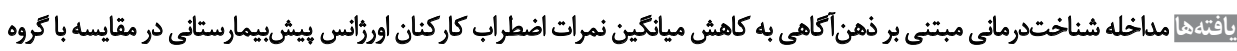

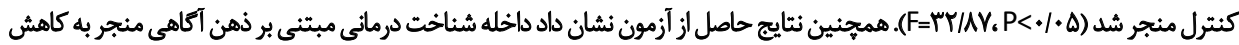

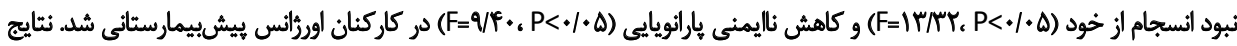

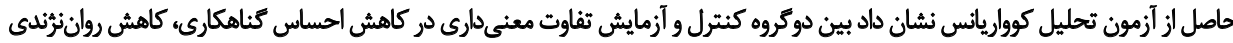

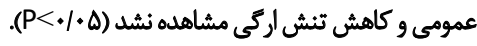

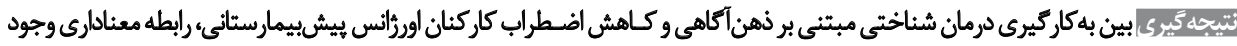

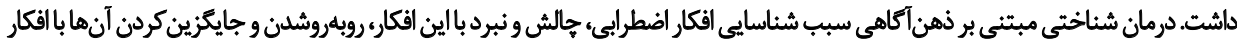

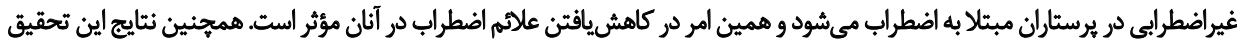

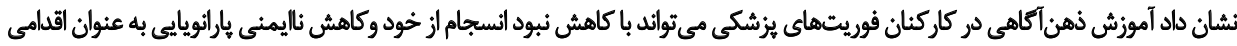

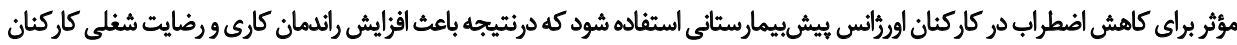

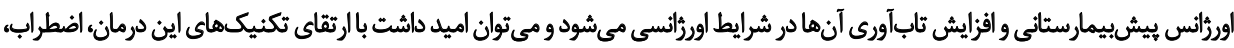

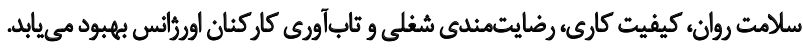

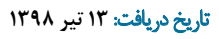

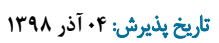

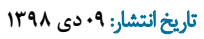


عملكرد شغلى و مشكلات جسمى و روانى، سبب فرسودگى

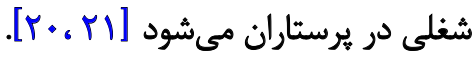

از جمله درمانهايى كه بهثازكى در روانشناسى و در حوزه

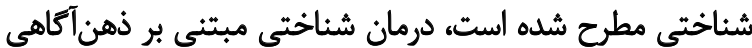

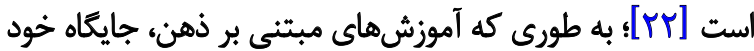

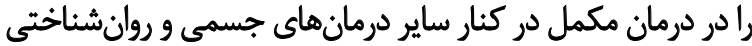

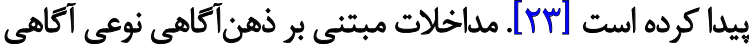

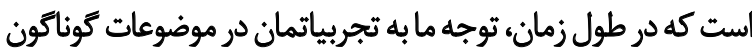

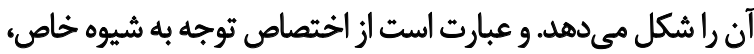

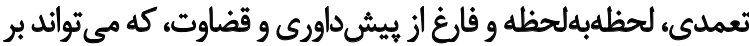

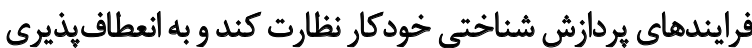

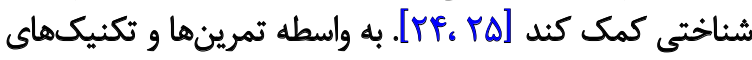

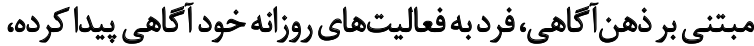

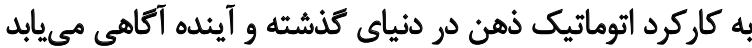

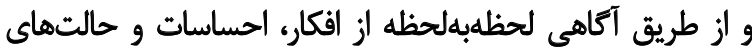

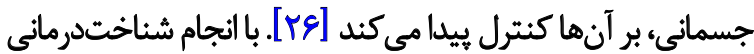

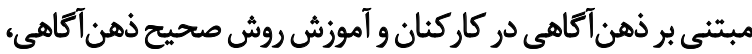

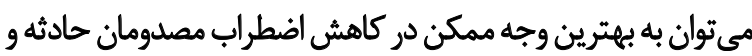

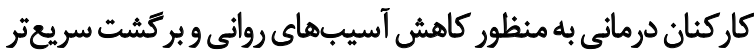
به روال عادى زندكى كمك كرفت [YrV]

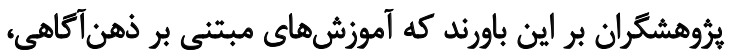

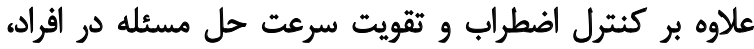

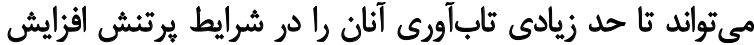

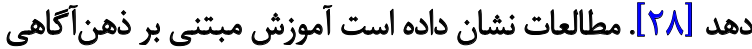

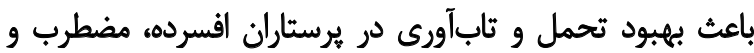

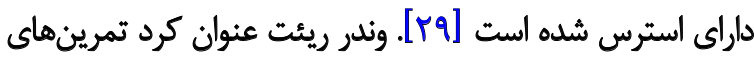

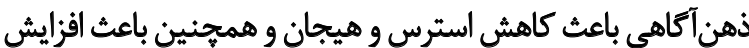

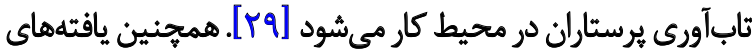

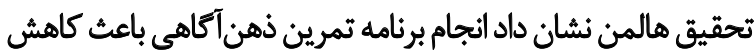

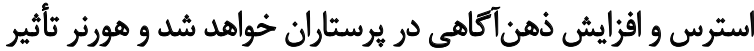

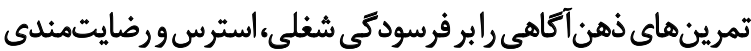

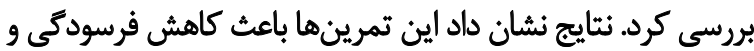

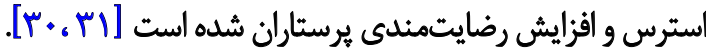

اضطراب و يُيامدهاى ناشى از آن، علاوه بر شكلدهى استرس

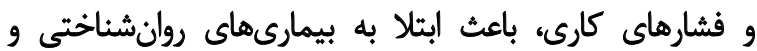

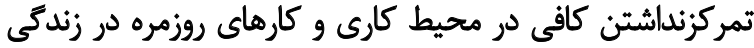

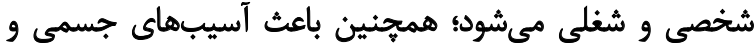

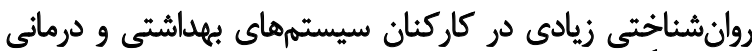

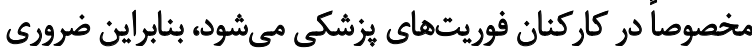

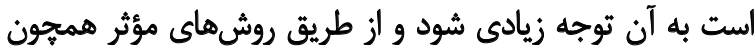

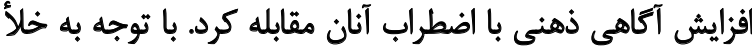

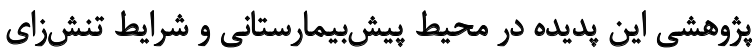
شغلى كاركنان فوريتهاى يزشكى، مطالعه حاضر با هدف بررسى مשlad

اضطراب به حالتى در فرد اشاره مى كند كه به موجب آن، وى

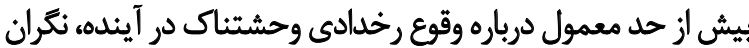

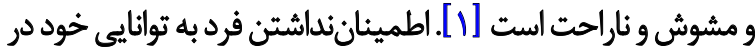

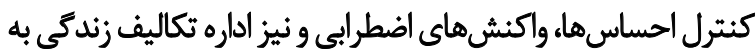

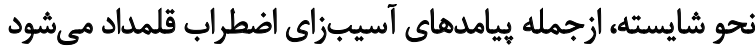

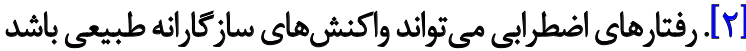
كه به فرد كمك كند تا واكنش مناسبيى در مقابله با شرايط سخت

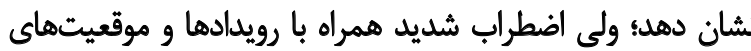

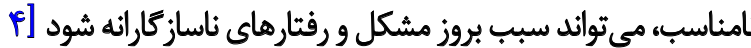

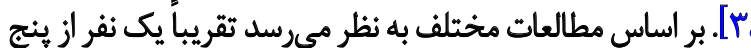

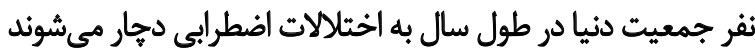

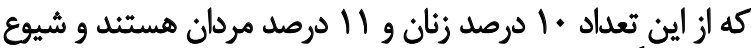

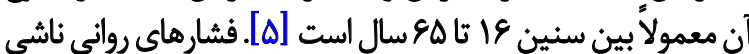

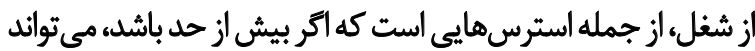

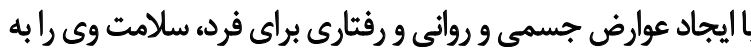

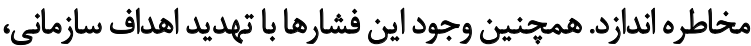

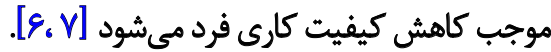

كاركنان بهداشتى و درمانى به دليل برخورد با بيماران مختلف،

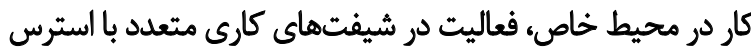

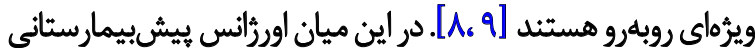

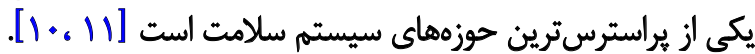

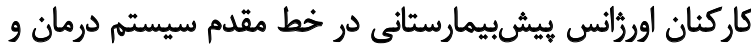

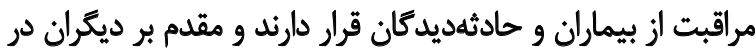

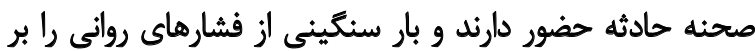

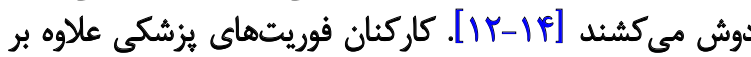

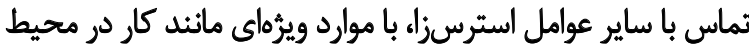

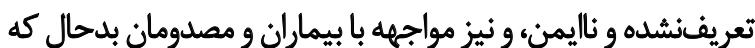

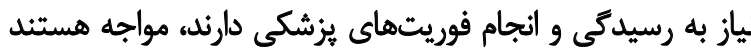

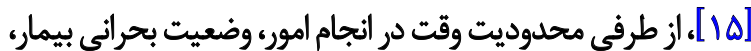

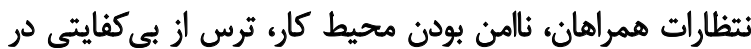

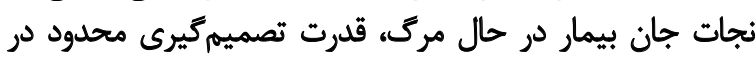

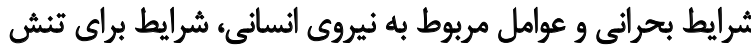

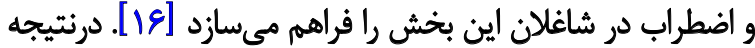

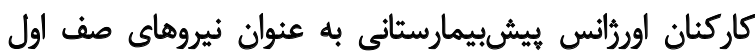

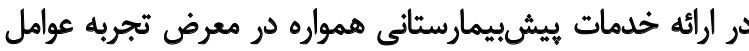

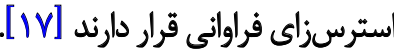

مطالعات متعددى ميزان بالاى استرس و اضطراب رادر بر بين

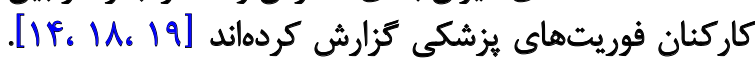

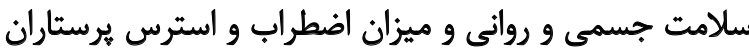

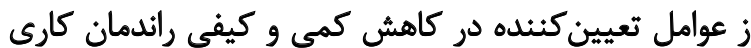

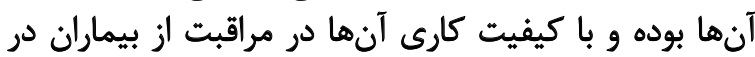

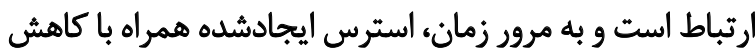


همكنى در متغيرهاى كتمى براى استفاده از آزمونهاى بارارمترى

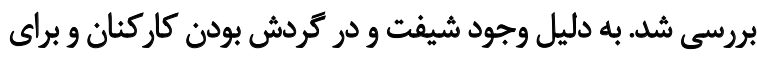

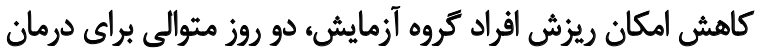
در نظر كرفته شد.

ابزار جمعآورى دادهها يرسشنامه اضطراب كتل بود. اين مقياس

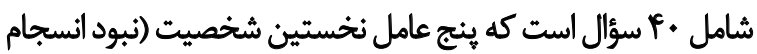

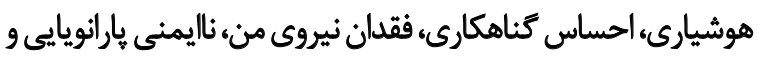

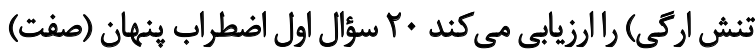

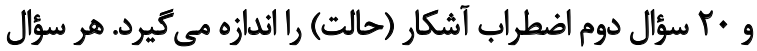

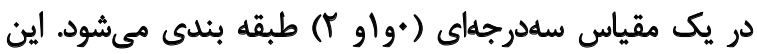

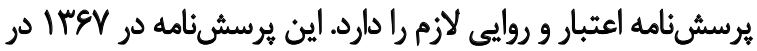

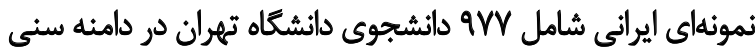

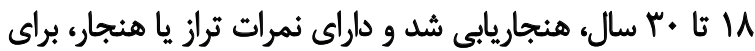

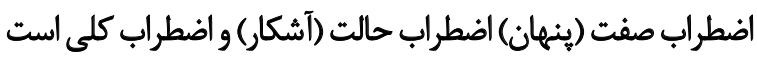

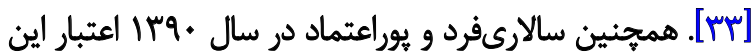

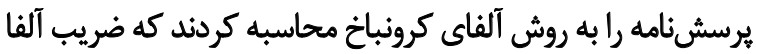

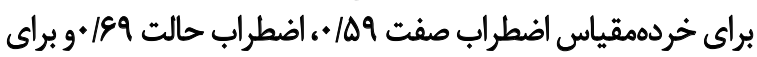

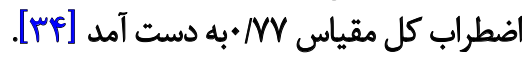
در تحقيق ديكرى فرامرزى، براي تعيين يايايي يرسشنامه اضطراب

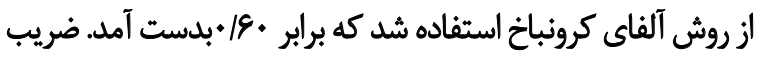

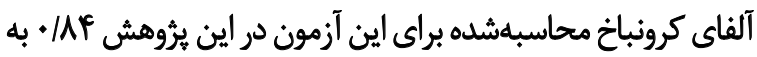

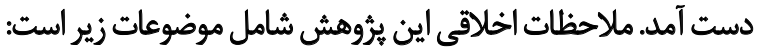

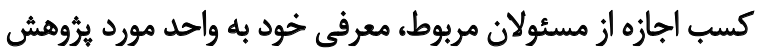

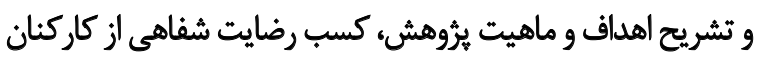

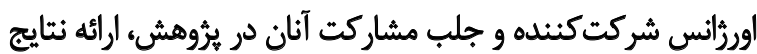

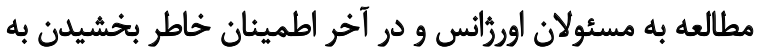

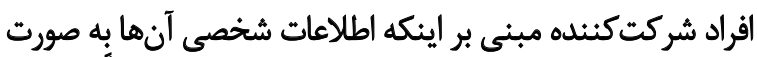

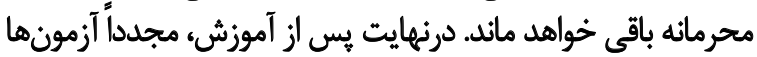

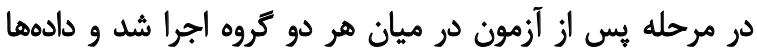

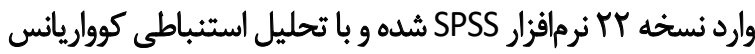
تجزيه و تحليل شد.

Latö

ميانكين و انحراف معيار سنى افراد شركتكنيده در مطالعه

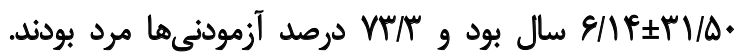

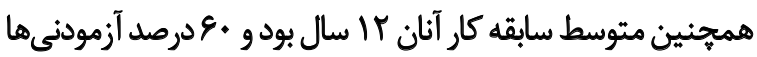

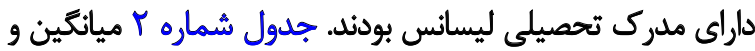

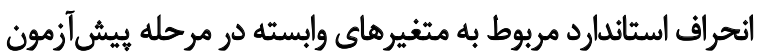

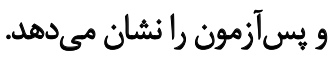

به منظور آزمون فرضيههاى يُوهش از تحليل كوواريانس

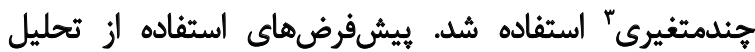

ميزان اثربخشى شئاختدرمانى مبتنى بر ذهن آثاهي بر كاهش

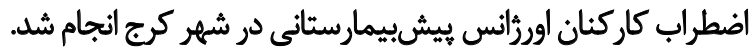

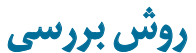

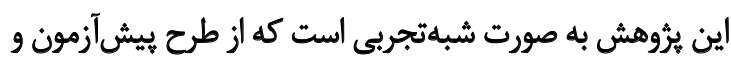

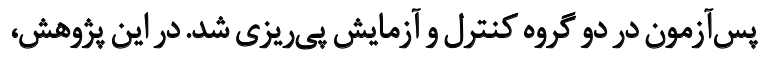

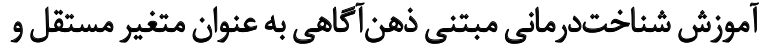

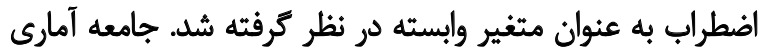

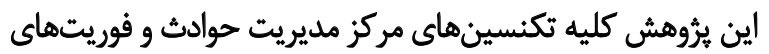

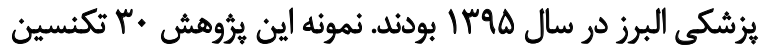

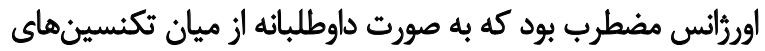

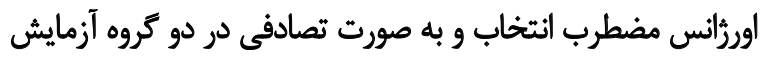

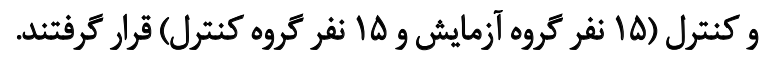

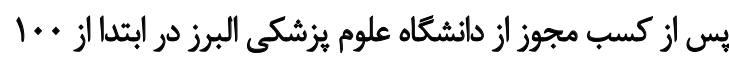

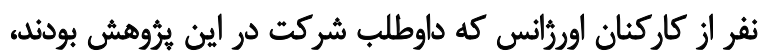

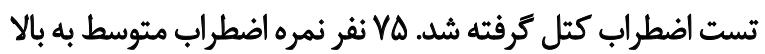

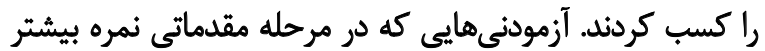

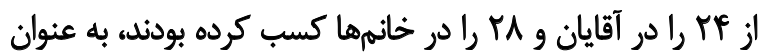

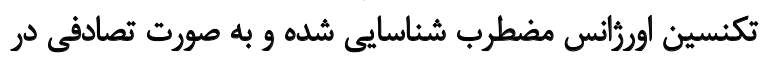

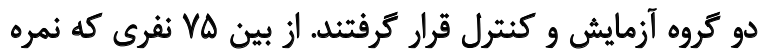

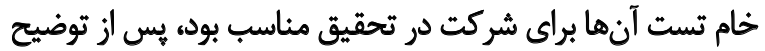

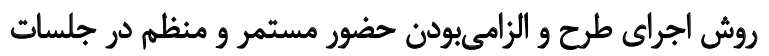

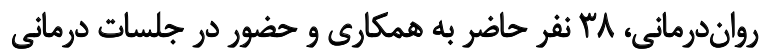

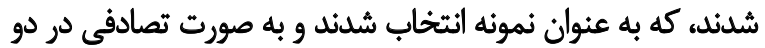

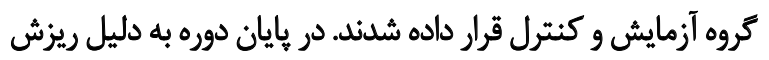

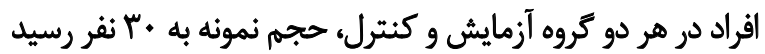

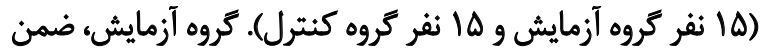

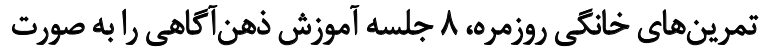

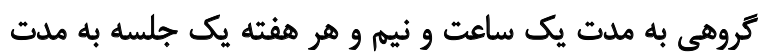

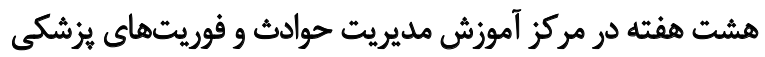

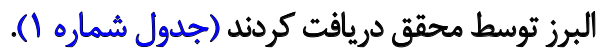

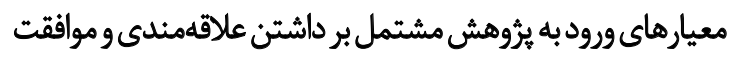

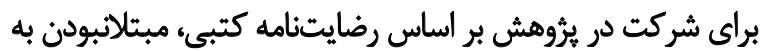

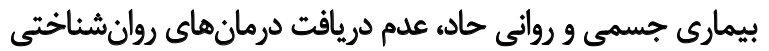

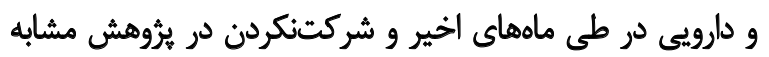

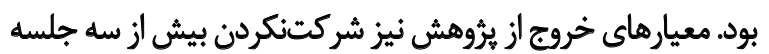

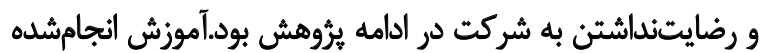

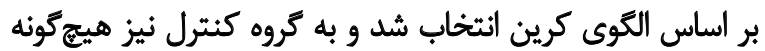

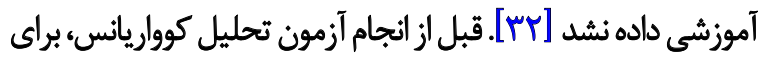

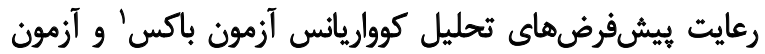

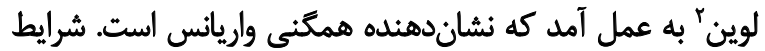

1. Box and Levene's tests

2.Levene's test of Equality of Error Variance 
جدول ا. خلاصه جدول آموزش شناختدرمانى مبتنى ذهن آكاهى

\begin{tabular}{|c|c|c|c|c|}
\hline تكليف خانكى & تغيير رفتار مورد انتظار & محتوا & هدف & شماره جلسه \\
\hline 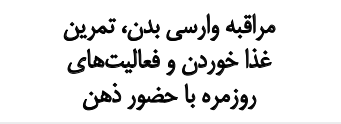 & 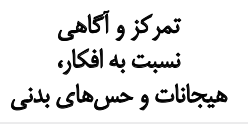 & 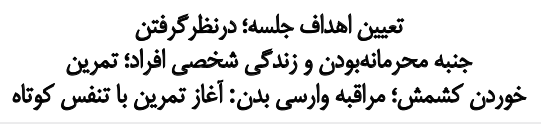 & 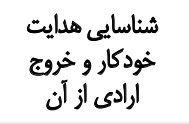 & اول ال \\
\hline 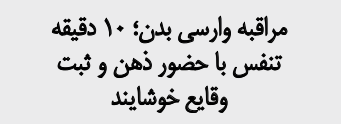 & 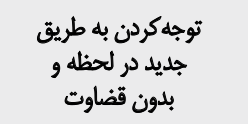 & ـا دقيقه تنفس وارسي بلنور ذهن & رويارويى با موانع & دوم \\
\hline 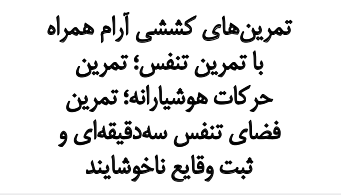 & 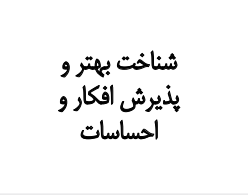 & 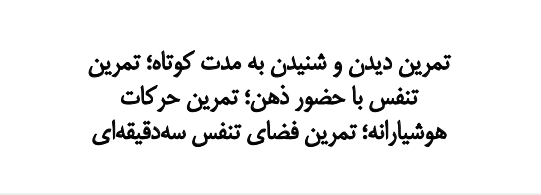 & 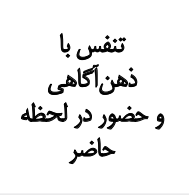 & سوم س \\
\hline 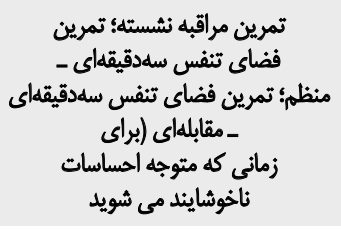 & تشخركز و كاهشى تشف & 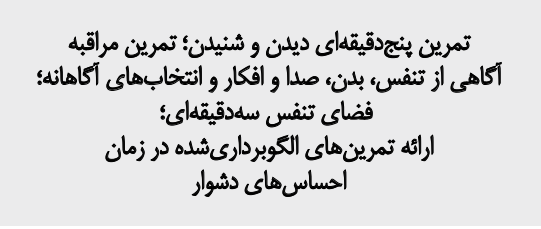 & ماندن در زمان حال & جهارم \\
\hline 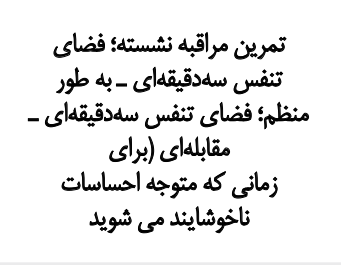 & 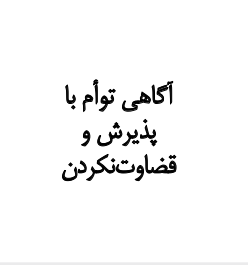 & 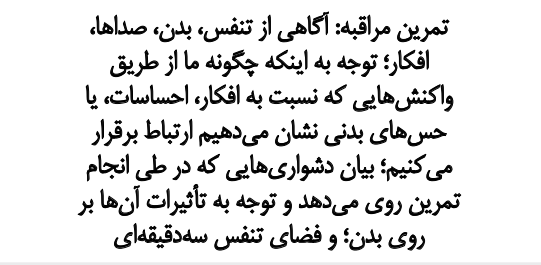 & يذيريرقتن و الجاز هدادن & بئجم \\
\hline 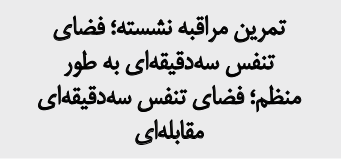 & 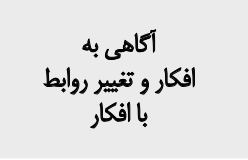 & 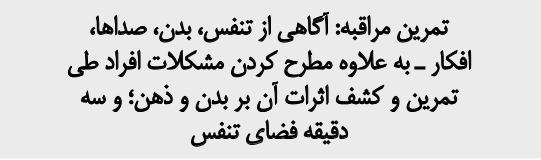 & افكار حقايق نيستند & 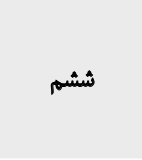 \\
\hline 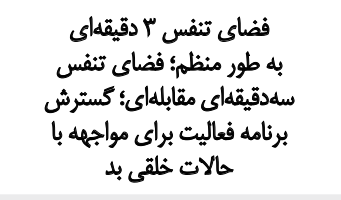 & 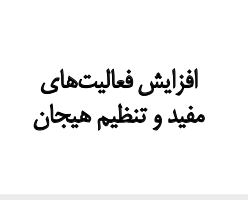 & 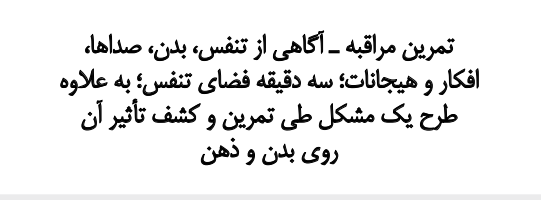 & 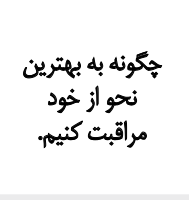 & هفتم \\
\hline الدامه تمرينهائ اصلى در زوزله & 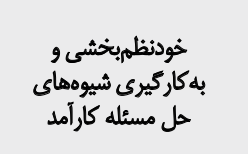 & مراقبه والرسى بلن، بهايايانرسائنن عراقبه & 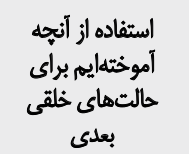 & هشتم \\
\hline
\end{tabular}

توانبخننى

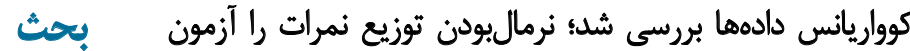

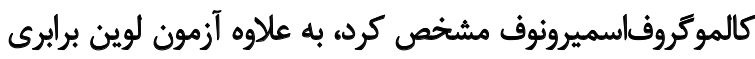
يافتههاى حاصل از ئروهش نشان داد بهاركيرى درمان

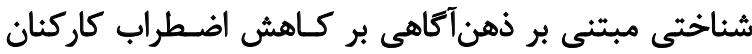

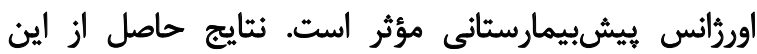

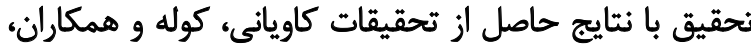

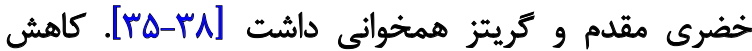

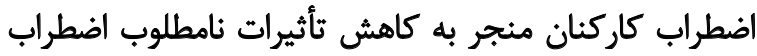

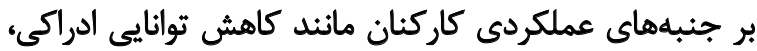

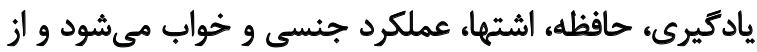

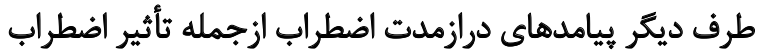

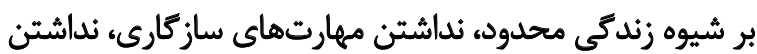

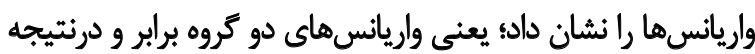

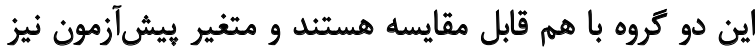

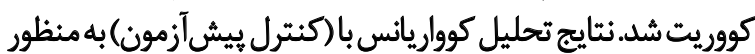

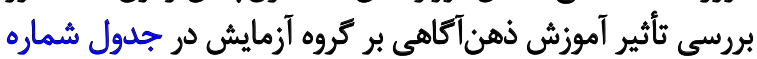

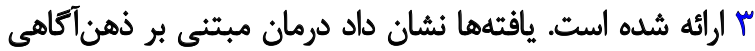

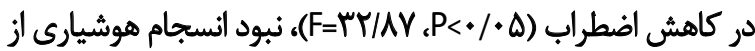

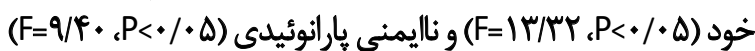

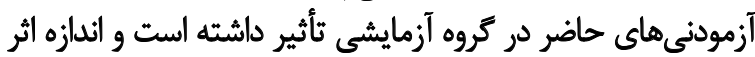

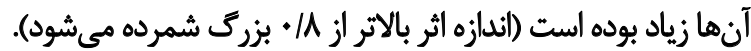


جدول ب. ميانكين و انحراف استاندارد مربوط به متغيرهاى وابسته در مرحله بيش آزمون و يس آزمون

\begin{tabular}{|c|c|c|c|c|}
\hline \multicolumn{4}{|c|}{ مياتكين||نحراف معيار } & \multirow{3}{*}{ متغير } \\
\hline \multicolumn{2}{|c|}{ كثترل } & \multicolumn{2}{|c|}{ آزمايش } & \\
\hline بسأزمون & ييش آزمون & يسأزمون & يبش أزمون & \\
\hline $12 / \pi \pm \pi / \Delta$ & $19 / T \Delta \pm \pm T / T \Delta$ & IV/PDET/AV & $r \Delta / T \backslash \pm Y / \Delta \Delta$ & اضطراب كلى \\
\hline $12 / m \pm 1 / V D$ & $19 / F \pm 1 / F T$ & $V / T \Delta \pm T / F T$ & $\mid 8 / 8 \cdot \pm r / .9$ & يود انسجام هوششيارى \\
\hline $\mid Q / \uparrow T \pm 1 / \& \Delta$ & $|\omega / \Delta \Delta \pm| / A^{C}$ & YNEPIVM & $r q / \circ E+r / Q$. & احساس كناهكارى \\
\hline$\| M \Psi \pm 1 / \Delta$ & $11 / 49 \pm 1 / \pi$ & V. $5 \pm 1 / 18$ & $11 / N \approx \pm 1 / V A$ & نبود نيروى من \\
\hline $1 .(9 \mathrm{~T} \pm 1 / 8$. & $1 . / 9 V \pm 1 / 16$ & $s / T \Delta \pm 1 / M$ & $1.188 \pm 1 / \% \Delta$ & ناليهنى يارالتويايى \\
\hline$r \cdot / r \cdot \pm l / M$ & $r+/ N+1 / / r$ & 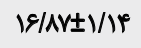 & $r+188 \pm 1 / 1 F$ & تش ارتى \\
\hline
\end{tabular}

توانبخننى

كاركنان مىشودو بر آن تأثير مستقيم و معنادارى دارد. نتايج حاصل

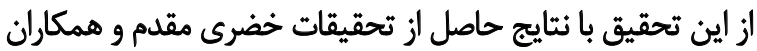

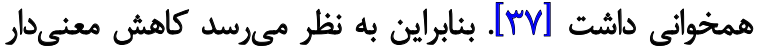

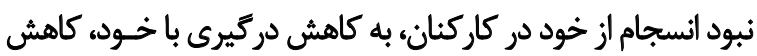

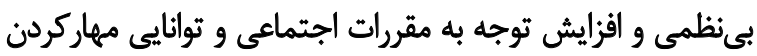

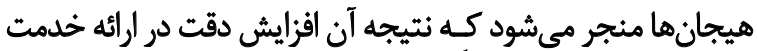

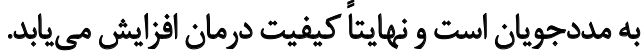

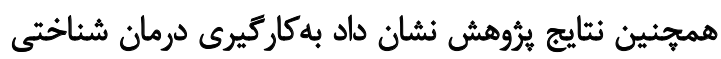

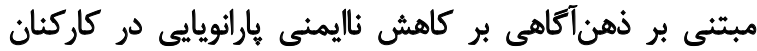

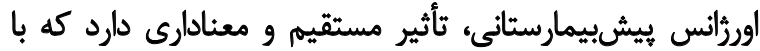

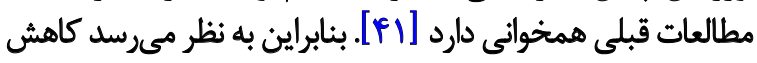

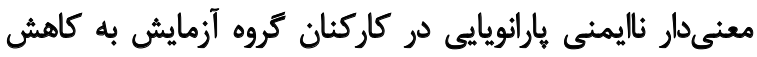

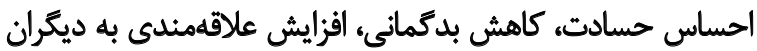

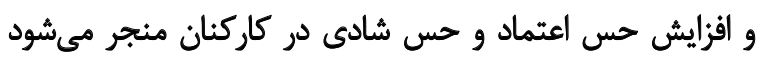

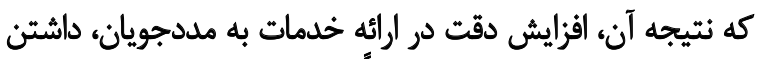

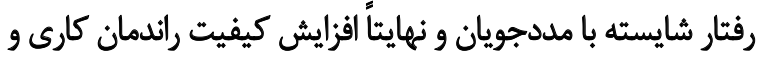
احساس رضايت شغلى در كاركنان مىشودي.
مهارتهاى اجتماعى، عزت نفس كم، احساس ناتوانى و روابط

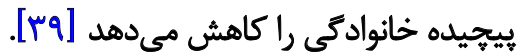
درباره اثربخشى درمان مبتنى بر آكاهى، بر كاهش اضطراب

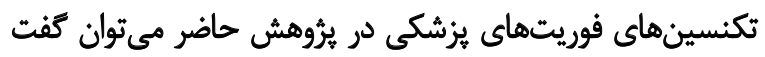

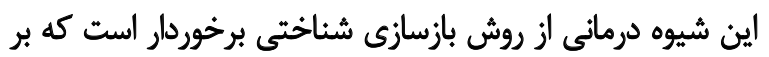

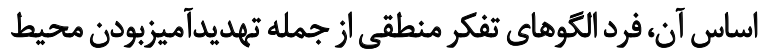

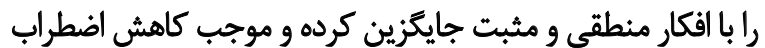

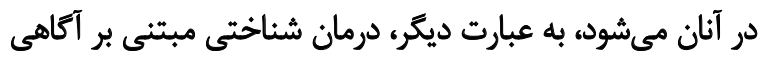

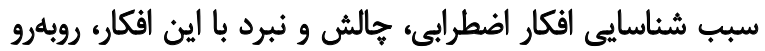

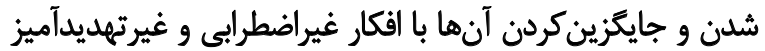

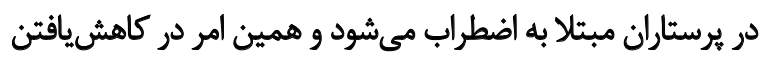

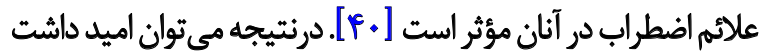

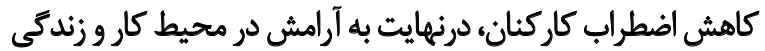
آنها منجر مىشود.

نتايج حاصل از آزمون نشان داد بهكاركيرى درمان شناختي مبتنى بر ذهن آكاهى منجر به كاهش نبود انسان انسجام هوشيارى در دران

جدول "ا. تحليل كوواريانس هندمتغيرى در دو كروه آزمايش و كثترل يس از تعديل نمرات ييشآزمون

\begin{tabular}{|c|c|c|c|c|c|c|c|}
\hline Effect Size & Eta & $\mathbf{P}$ & $\mathbf{F}$ & MS & df & SS & متغير \\
\hline$V / N$ & - MAS & .0 .4 & Tr/Av & ITT/TVE & 1 & ITT/TVE & اضطراب \\
\hline \multirow[t]{4}{*}{ M/r } & .1890 & .1 .11 & I & $1 .+1 .+.5$ & 1 & $1 .+\% / .+4$ & نبود اتسجام هوشيارى از خود \\
\hline & - &.$/ N H$ & T/MAT & $P / N$. & 1 & YME. & كرايش به احساس كناهكارى \\
\hline & - & +1.97 & F/IAT & PV/aAq & 1 & PV/QDQ & تنش اركى \\
\hline & - & $+/ \triangle A F$ & r/AYA & $r / q)$. & 1 & $p / q 1$. & تيود ثيروى من \\
\hline.$/ Q 8$ &.$/ \pi T$ & .10 .9 & $9 / 4+4$ & IF/FIV & 1 & IF/FIV & ناليمنى يارانوئيدى \\
\hline
\end{tabular}

توانبخننى 
در شرايط اوررأنسى ارتقا يابد. بيشنهاد مي شئود مسئولان سازمان

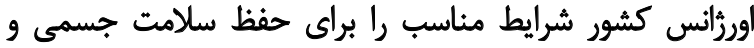

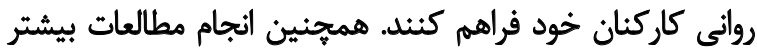

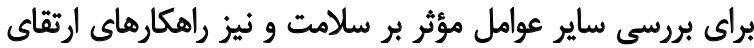

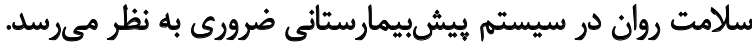

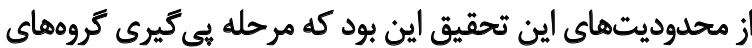

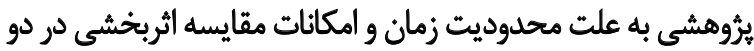

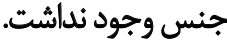

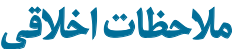

\section{بيروى أز اصول الخالق يثوهش}

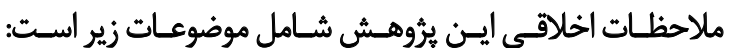

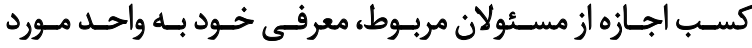

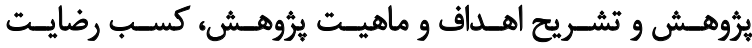

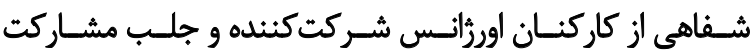

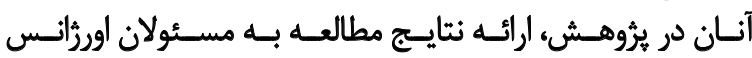

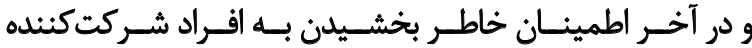

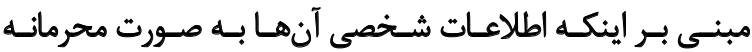

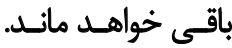

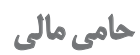

اين مقاله، مستخرج از ياياننامه كارشناسىارشد نويسنده دوم، در كروه روانشناسى بالينى دانشعاه آزاد كرمان است.

مشاركت نويسندكان

مفهومسازى، تحقيق و بررسى:صابرىنياو افلاطونيان و امينىزاده؛ ويراستارى و نهاييىازىى امينىزاده، جنت و وابرىىنيا.

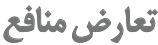

بنابر اظهار نويسندكان، اين مقاله تعارض منافع ندارد.
از طرفى ديكر يافتهاي تحقيق نشان مى دهد به كاركيرى درمان

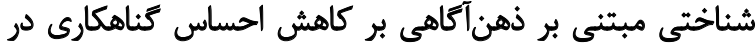

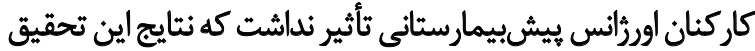

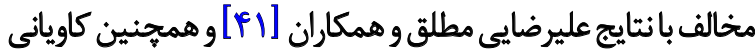

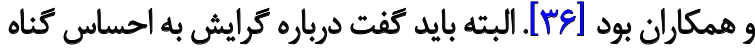

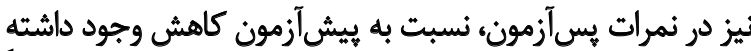

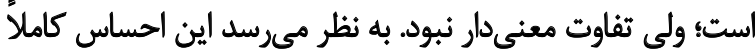

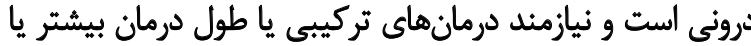

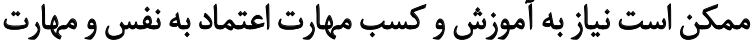

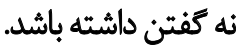

نتايج حاصل از يافتههاى تحقيق نشان داد بهكاركيرى درمان

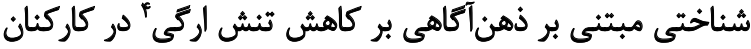

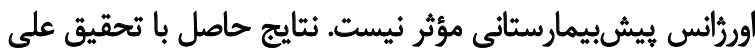

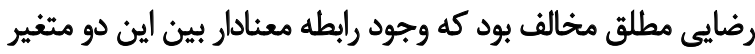

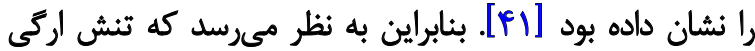

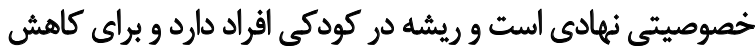

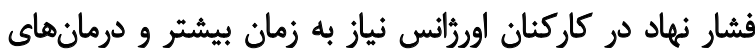
تركيبى، كاهى دارويى و حتى تكيه بر جلسات تكنيفره دارد. درنهايت بين بهكاركيرى درمان شناختى مبتنى بر ذهن آحاهي با

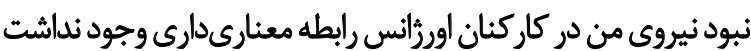

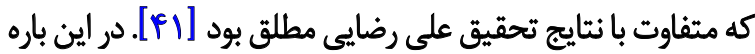

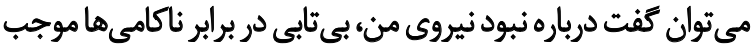

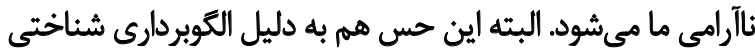

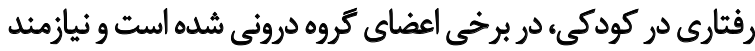

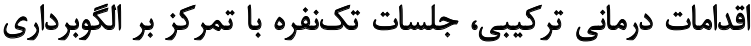

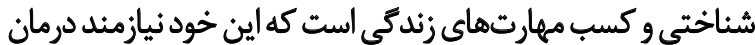

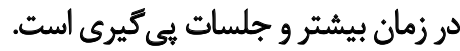
تشيجهي بافه طور كلى اين يثروهش در همسويى با تحقيقات بيشين نشان داد

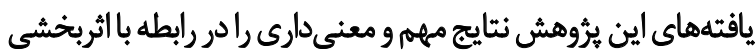

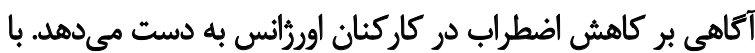

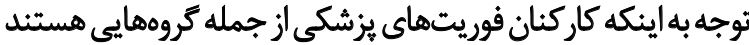

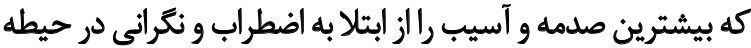

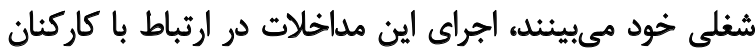

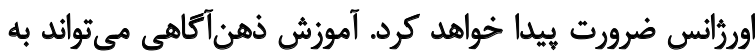

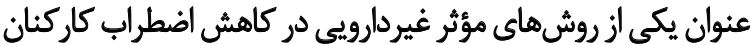

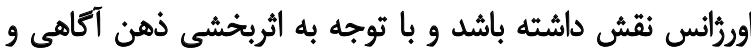

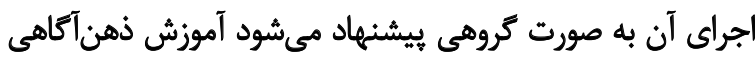

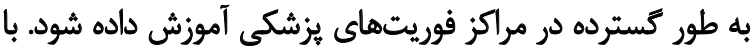

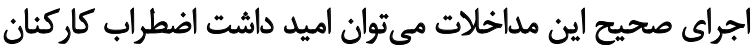

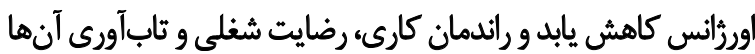

4. Ergic or drive tension 


\section{References}

[1] Hancock KM, Swain J, Hainsworth CJ, Dixon AL, Koo S, Munro K. Acceptance and commitment therapy versus cognitive behavior therapy for children with anxiety: Outcomes of a randomized controlled trial. Journal of Clinical Child \& Adolescent Psychology. 2018; 47(2):296-311. [DOI:10.1080/15374416.2015.111082 2] [PMID]

[2] Hofmann SG, Sawyer AT, Witt AA, Oh D. The effect of mindfulness-based therapy on anxiety and depression: A meta-analytic review. Journal of Consulting and Clinical Psychology. 2010; 78(2):169-83. [DOI:10.1037/a0018555] [PMID] [PMCID]

[3] Hollocks MJ, Lerh JW, Magiati I, Meiser-Stedman R, Brugha TS. Anxiety and depression in adults with autism spectrum disorder: A systematic review and meta-analysis. Psychological Medicine. 2019; 49(4):559-72. [DOI:10.1017/S0033291718002283] [PMID]

[4] Ferrazzo S, Gunduz-Cinar O, Stefanova N, Pollack GA, Holmes A, Schmuckermair C, et al. Increased anxiety-like behavior following circuit-specific catecholamine denervation in mice. Neurobiology of Disease. 2019; 125:55-66. [DOI:10.1016/j.nbd.2019.01.009] [PMID]

[5] Abolghasemi A, Asadi S. [Assessment of the efficacy of mindfulness-based cognitive therapy on emotional processing in anxious nurses (Persian)]. Journal of Nursing Education. 2016; 5(3):17-24. [DOI:10.21859/jne-05033]

[6] Rahmani F, Behshid M, Zamanzadeh V, Rahmani F. [Relationship between general health, occupational stress and burnout in critical care nurses of Tabriz Teaching Hospitals (Persian)]. Iran Journal of Nursing. 2010; 23(66):54-63.

[7] Poursadeghiyan M, Abbasi M, Mehri A, Hami M, Raei M, Ebrahimi MH. Relationship between job stress and anxiety, depression and job satisfaction in nurses in Iran. The Social Sciences. 2016; 11(9):2349-55. [DOI:10.3923/sscience.2016.2349.2355]

[8] Aazami Y, Sohrabi F, Borjali A, Farrokhi N, Farokh Forghani S. The effectiveness of psychosocial model-based therapy on social skills in people With PTSD after burn. Archives of Rehabilitation. 2018; 19(3):206-19. [DOI:10.32598/ri.19.3.206]

[9] Ghaddar A, Mateo I, Sanchez P. Occupational stress and mental health among correctional officers: A cross-sectional study. Journal of Occupational Health. 2008; 50(1):92-8. [DOI:10.1539/ joh.50.92] [PMID]

[10] Aminizade M, Nekouei Moghaddam M, Birami Jam M, Shamsi $\mathrm{M}$, Majidi $\mathrm{N}$, Amanat $\mathrm{N}$, et al . The role of volunteer citizens in response to accidents and disasters. Health in Emergencies and Disasters. 2017; 2(3):107-24 [DOI:10.18869/nrip.hdq.2.3.107]

[11] Sheykhbardsiri H, Amini Zadeh M, Vali L, Esmaeli MA, Amini zadeh Gh, Amini Zadeh A. [The relationship between the quality of worklife and organizational commitment among personnel of emergency medical services (Persian)]. Journal of Management and Medical Informatics School. 2013; 1(1):38-46.

[12] Sepahvand E, Khankeh HR, Ebadi A. Psychometric properties of the Persian version of readiness for events with psychological emergencies assessment tool. Health in Emergencies and Disasters Quarterly. 2017; 2(4):207-16. [DOI:10.29252/nrip. hdq.2.4.207]
[13] Yarmohammadi H, Pourmohammadi A, Sohrabi Y, Eskandari S, Poursadeghiyan $\mathrm{M}$, Biglari $\mathrm{H}$, et al. Work shift and its effect on nurses' health and welfare. Social Sciences (Pakistan). 2016; 11(9):2337-41.

[14] Aminizadeh M, Rasouli Ghahfarokhi SM, Pourvakhshoori N, Beyramijam M, Majidi N, Shahabi Rabori MA. Comparing the effects of two different educational methods on clinical skills of emergency intermediate technician: A quasi-experimental research. Journal of Education and Health Promotion. 2019; 8:54. [PMID] [PMCID]

[15] Abbasi M, Farhang Dehghan S, Fallah Madvari R, Mehri A, Ebrahimi $\mathrm{MH}$, Poursadeghiyan $\mathrm{M}$, et al. Interactive effect of background variables and workload parameters on the quality of life among nurses working in highly complex hospital units: A crosssectional study. Journal of Clinical and Diagnostic Research. 2019; 13(1):LC8-LC13. [DOI:10.7860/JCDR/2019/37929.12482]

[16] Vali L, Amini Zade M, Sharifi T, Oroomiei N, Mirzaee S. [Assessing job stress factors among medical emergency technicians 115 of Kerman (Persian)]. Journal of Hospital. 2014; 13(2):75-85.

[17] Aminizadeh M, Tirgari B, Iranmanesh S, Garoosi B, Karimi M Sheikh-Bardsiri H. [Post-traumatic stress disorder prevalence in employees of emergency departments of teaching hospitals affiliated to Kerman University of Medical Sciences, Iran (Persian)] Journal of Management and Medical Informatics School. 2013 $1(1): 18-25$

[18] França SP, De Martino MM, Aniceto EV, Silva LL. Predictors of burnout syndrome in nurses in the prehospital emergency services. Acta Paulista de Enfermagem. 2012; 25(1):68-73. [DOI:10.1590/S0103-21002012000100012]

[19] Bentley MA, Crawford JM, Wilkins JR, Fernandez AR, Studnek JR. An assessment of depression, anxiety, and stress among nationally certified EMS professionals. Prehospital emergency Care. 2013; 17(3):330-8. [DOI:10.3109/10903127.2012.761307] [PMID]

[20] Aminizadeh M, Eslamishahr Babaki M, Beyramijam M, Aminizadeh $M$, Sheikhbardsiri H. Prioritizing motivational and satisfactorily factors of volunteer medical and health personnel in natural disasters. Health in Emergencies \& Disasters Quarterly. 2016; 1(2):79-88

[21] Mirzaei M, Sepahvand E. Evaluation of the occupational stress and the effective factors on it in the staff of educational hospital of Shohada-e-Ashayer of Khoram Abad in 2014. Journal of Dental and Medical Sciences. 2015; 14(9):80-4.

[22] Dahlgaard J, Jørgensen MM, van der Velden AM, Sumbundu A, Gregersen N, Olsen RK, et al. Mindfulness, health, and longevity. In: Rattan SIS, Kyriazis M, editors. The Science of Hormesis in Health and Longevity. Cambridge: Academic Press; 2019. [DOI:10.1016/B978-0-12-814253-0.00022-X]

[23] Lindsay EK, Young Sh, Smyth JM, Brown KW, Creswell JD. Acceptance lowers stress reactivity: Dismantling mindfulness training in a randomized controlled trial. Psychoneuroendocrinology. 2018; 87:63-73. [DOI:10.1016/j.psyneuen.2017.09.015] [PMID]

[24] Eby LT, Allen TD, Conley KM, Williamson RL, Henderson TG, Mancini VS. Mindfulness-based training interventions for employees: A qualitative review of the literature. Human Resource Management Review. 2019; 29(2):156-78. [DOI:10.1016/j. hrmr.2017.03.004] 
[25] Rashidian A, Karbalaei Nouri A, Hosseinzadeh S, Haghgoo H. [The Bay Area Functional Performance Evaluation-Task Oriented Assessment (BaFPE-TOA) in severe psychiatric patients: A psychometric study (Persian)]. Archives of Rehabilitation. 2018; 19(2):168-77. [DOI:10.32598/rj.19.2.168]

[26] MacKenzie MB, Kocovski NL. Mindfulness-based cognitive therapy for depression: Trends and developments. Psychology Research and Behavior Management. 2016; 9:125-32. [DOI:10.2147/PRBM.S63949] [PMID] [PMCID]

[27] Teasdale JD, Segal ZV, Williams JMG, Ridgeway VA, Soulsby $\mathrm{JM}$, Lau MA. Prevention of relapse/recurrence in major depression by mindfulness-based cognitive therapy. Journal of Consulting and Clinical Psychology. 2000; 68(4):615-23. [DOI:10.1037//0022-006X.68.4.615] [PMID]

[28] Foureur M, Besley K, Burton G, Yu N, Crisp J. Enhancing the resilience of nurses and midwives: Pilot of a mindfulnessbased program for increased health, sense of coherence and decreased depression, anxiety and stress. Contemporary Nurse. 2013; 45(1):114-25. [DOI:10.5172/conu.2013.45.1.114] [PMID]

[29] van der Riet P, Levett-Jones T, Aquino-Russell C. The effectiveness of mindfulness meditation for nurses and nursing students: An integrated literature review. Nurse Education Today. 2018; 65:201-11. [DOI:10.1016/j.nedt.2018.03.018] [PMID]

[30] Hallman IS, O'Connor N, Hasenau S, Brady S. Improving the culture of safety on a high-acuity inpatient child/adolescent psychiatric unit by mindfulness-based stress reduction training of staff. Journal of Child and Adolescent Psychiatric Nursing. 2014; 27(4):183-9. [DOI:10.1111/jcap.12091] [PMID]

[31] Horner JK, Piercy BS, Eure L, Woodard EK. A pilot study to evaluate mindfulness as a strategy to improve inpatient nurse and patient experiences. Applied Nursing Research. 2014 27(3):198-201. [DOI:10.1016/j.apnr.2014.01.003] [PMID]

[32] Crane RS, Kuyken W, Hastings RP, Rothwell N, Williams JM. Training teachers to deliver mindfulness-based interventions: Learning from the UK experience. Mindfulness. 2010; 1(2):7486. [DOI:10.1007/s12671-010-0010-9] [PMID] [PMCID]

[33] Carpenter JK, Sanford J, Hofmann SG. The effect of a brief mindfulness training on distress tolerance and stress reactivity. Behavior Therapy. 2019; 50(3):630-45. [DOI:10.1016/j. beth.2018.10.003] [PMID]

[34] Salarifar MH, Pouretemad HR. [The relationship between metacognitive beliefs and anxiety and depression disorder (Persian)]. Yafte. 2012; 13(4):29-38.

[35] Kaviani H, Hatami N, Javaheri F. The impact of Mindfulnessbased Cognitive Therapy (MBCT) on mental health and quality of life in a sub-clinically depressed population. Archives of Psychiatry and Psychotherapy. 2012; 14(1):21-8.

[36] Flugel Colle KF, Vincent A, Cha SS, Loehrer LL, Bauer BA, Wahner-Roedler DL. Measurement of quality of life and participant experience with the mindfulness-based stress reduction program. Complementary Therapies in Clinical Practice. 2010; 16(1):36-40. [DOI:10.1016/j.ctcp.2009.06.008] [PMID]

[37] Khezri Moghadam N, Vahidi S, Ashormahani M. Efficiency of cognitive-existential group therapy on life expectancy and depres- sion of elderly residing in nursing home. Iranian Journal of Ageing. 2018; 13(1):62-73. [DOI:10.21859/sija.13.1.62]

[38] Gratz KL, Gunderson JG. Preliminary data on an acceptancebased emotion regulation group intervention for deliberate self-harm among women with borderline personality disorder. Behavior Therapy. 2006; 37(1):25-35. [DOI:10.1016/j. beth.2005.03.002] [PMID]

[39] Ma SH, Teasdale JD. Mindfulness-based cognitive therapy for depression: replication and exploration of differential relapse prevention effects. Journal of Consulting and Clinical Psychology. 2004; 72(1):31-40. [DOI:10.1037/0022-006X.72.1.31] [PMID]

[40] Khoury B, Lecomte T, Fortin G, Masse M, Therien P, Bouchard V, et al. Mindfulness-based therapy: A comprehensive meta-analysis. Clinical Psychology Review. 2013; 33(6):763-71. [DOI:10.1016/j. cpr.2013.05.005] [PMID]

[41] Alirezai Motlaq M, Asadi Z. [Cognitive-Behavioral Therapy (CBT) in ameliorating adolescent anxiety in Generalized Anxiety Disorders (GAD) (Persian)]. Journal of Exceptional Children. 2009; 9(1):25-34 
This Page Intentionally Left Blank 